Article

\title{
Preparation, Characterization, and In Vivo Pharmacokinetic Evaluation of Polyvinyl Alcohol and Polyvinyl Pyrrolidone Blended Hydrogels for Transdermal Delivery of Donepezil HCl
}

\author{
Santosh Bashyal ${ }^{1}$, Chang Yell Shin ${ }^{2}$, Sang Min Hyun ${ }^{2}$, Sun Woo Jang ${ }^{2, *}$ and Sangkil Lee ${ }^{1, *}$ \\ 1 College of Pharmacy, Keimyung University, 1095 Dalgubeol-daero, Dalseo-gu, Daegu 42601, Korea; \\ bashyal.santosh18@gmail.com \\ 2 Research Institute of Dong-A ST Co. Ltd., Yongin 17073, Korea; pharm91@donga.co.kr (C.Y.S.); \\ hsm19@donga.co.kr (S.M.H.) \\ * Correspondence: chb@donga.co.kr (S.W.J.); skdavid@kmu.ac.kr (S.L.); Tel.: +82-31-280-1324 (S.W.J.); \\ +82-53-580-6655 (S.L.); Fax: +82-31-282-8564 (S.W.J.); +82-53-580-5164 (S.L.)
}

Received: 20 February 2020; Accepted: 12 March 2020; Published: 16 March 2020

\begin{abstract}
Transdermal delivery systems are emerging platforms for the delivery of donepezil hydrochloride (DH) for treating Alzheimer's disease. The primary aim of this study was to develop polyvinyl alcohol and polyvinyl pyrrolidone blended hydrogels and to evaluate their feasibility for delivering DH via a transdermal route. Physicochemical properties, such as gel fraction (\%), swelling ratio (\%), weight loss (\%), mechanical strength, elongation at break, and Young's modulus of the prepared hydrogels were evaluated. Furthermore, in vitro skin permeation and in vivo pharmacokinetic studies were performed. With an increased concentration of propylene glycol (PG), the gel fraction (\%), maximum strength, and elongation at break decreased. However, the swelling ratio (\%) and weight loss (\%) of hydrogels increased with increased PG content. The 26\% PG-hydrogel was superior, with an enhancement ratio of $\left.12.9{ }^{* * *} p<0.001\right)$. In addition, the $11 \%$ PG-hydrogel and $1 \%$ PG-hydrogel exhibited an enhancement ratio 6.30-fold ( $\left.{ }^{* * *} p<0.001\right)$ and 2.85 -fold $\left({ }^{*} p<0.05\right)$ higher than that exhibited by control, respectively, indicating a promising effect of PG on skin permeation. In addition, in vivo pharmacokinetic studies on hairless rats assessed the expediency for transdermal delivery of DH. The transdermal delivery of optimized hydrogel-patches with two different doses of $\mathrm{DH}$ revealed that the maximum plasma concentration and area under the curve were dose dependent, and the time to reach the maximum concentration was $8 \mathrm{~h}$. Thus, optimized hydrogels have the potential to enhance the transdermal delivery of DH and could be a novel clinical approach.
\end{abstract}

Keywords: transdermal delivery; hydrogel; skin permeation; donepezil; propylene glycol; chemical enhancer

\section{Introduction}

Alzheimer's disease (AD) is a chronic neurodegenerative disorder that causes dementia [1]. In 2015, 46.8 million people were affected by $\mathrm{AD}$, and this prevalence is estimated to increase to 75 million and 131.5 million globally by 2030 and 2050, respectively [2]. There are approximately 5.8 million Americans with AD in 2019 [3]. Due to the impairment of cholinergic neurotransmission, there is a decreased production of the neurotransmitter acetylcholine, leading to AD [4]. Donepezil hydrochloride (DH) is the second well-tolerated FDA-approved medication for the treatment of AD [5]. DH is a centrally acting reversible acetylcholinesterase inhibitor that decreases the availability of beta amyloid and 
increases the concentration of acetylcholine [6,7]. DH is administered to patients orally; however, significant variations in DH plasma concentrations and gastrointestinal side-effects, including nausea, vomiting, anorexia, and abdominal pain, are associated with oral administration. In addition, owing to dementia and dysphagia, elderly patients have difficult in consuming medicines effectively [8,9]. Thus, transdermal drug delivery systems (TDDSs) can circumvent issues associated with oral administration and may be the best therapeutic platform for improving patient compliance.

TDDS offers a multitude of advantages over oral administration. Owing to the maintenance of constant plasma levels over a long period, the dosing frequency is attenuated and bioavailability of the drug across the skin is improved. Additionally, the first-pass effect and gastrointestinal discomfort can be avoided. Patients can easily terminate the treatment, which improves patient compliance [10-12]. The molecular structure and essential properties of DH are summarized in Table 1. All parameters required to increase lipophilicity and enhance drug permeability, such as molecular weight, H-bond donation, H-bond acceptance, and the $\log \mathrm{P}$ value of DH, follow the Rule of Five. However, owing to the presence of a permeability barrier (the stratum corneum) in the skin, formulating a TDDS of DH is still a major concern for researchers $[13,14]$. Chemical permeability enhancers offer tremendous potential to overcome this limitation. In general, chemical enhancers facilitate the transfer of drug across skin layers mainly by two mechanisms: by extract of lipids directly from the skin (lipid extraction) and by partitioning into lipid bilayers (lipid fluidization) [15].

Table 1. Molecular structure and essential properties of donepezil hydrochloride (DH).

\begin{tabular}{c}
\hline Molecular Structure \\
\hline Molecular weight \\
\hline H-bond donor
\end{tabular}

In this study, we used propylene glycol (PG) as the primary chemical permeation enhancer for facilitating DH permeability across the skin, both in vitro and in vivo. PG is reportedly a safe and effective chemical permeability enhancer across the skin [16]. PG is listed as generally regarded as safe by the United States Food and Drug Administration (FDA). In addition, the World Health Organization has reported that the acceptable daily intake of PG is up to $25 \mathrm{mg} / \mathrm{kg}$ body weight [17]. The mechanism of PG that facilitates drug permeability is not clearly understood. However, it has been proposed that PG acts by solvating the keratin present in the stratum corneum and intercalating the polar head groups of lipid bilayers [18].

Hydrogels are cross-linked three-dimensional hydrophilic polymeric networks and have the potential to retain substantial amounts of aqueous fluids or water $[19,20]$. Hydrogels have been widely explored for numerous biomedical applications, such as in drug delivery, soft contact lenses, biomaterials, artificial organs, superabsorbent implants, stimuli-responsive systems, wound dressings, or tissue engineering, owing to their excellent biocompatibility, biodegradability, non-toxicity, non-antigenicity, elasticity, high water content, and soft consistency [21,22]. These characteristics render hydrogels ideal for transdermal applications. The choice of polymers used to fabricate hydrogels has a significant impact on swelling, elasticity, and mechanical properties, as well as skin permeation of the drug. It was previously reported that poly(vinyl alcohol) (PVA) and poly(vinyl pyrrolidone) 
(PVP) are easily blended and cross-linked to form desirable hydrogels [23,24]. In addition, hydroxyl group of PVA will interact with carbonyl group of PVP leading to the formation of interchain of hydrogen bonding and enhances the network stability [25]. The drug will be located across this polymer network. However, the addition of cosolvent will reduces the cross-linking density and network formation resulting in the enhanced elasticity and flexibility of hydrogels. Moreover, cosolvent will engross the water molecules from the release medium, and increases the solubilization of the drug, leading to decreasing the viscosity of the hydrogels and increasing the release rate of the drug [26]. These PVA-PVP blended hydrogels have improved water uptake capacity, possess high elasticity and soft consistency, are pleasant to touch, terminate easily without pain, and have mechanical strength and transparency [27]. In this study, physical cross-linking techniques were adopted to cross-link PVA-PVP blended hydrogels owing to their superior qualities, including non-toxicity and higher mechanical strength, over chemical or irradiative methods [28].

The main objective of this study was to fabricate, optimize, and characterize PVA-PVP blended hydrogels for transdermal delivery of $\mathrm{DH}$. In this perspective, the gel fraction (\%), swelling ratio $(\%)$, weight loss $(\%)$, and mechanical properties of the developed hydrogels were evaluated, and the effect of PG on skin permeation of DH was assessed. Finally, in vivo pharmacokinetic studies were conducted using hairless rats to assess the expediency for transdermal delivery of DH.

\section{Materials and Methods}

\subsection{Materials}

DH was provided by the Research Institute of Dong-A ST Co. Ltd. (Yongin, Korea). PVP K-90, PVP K-30, and PG were purchased from BASF (Ludwigshafen, Germany). PVA and glycerol were purchased from Sigma-Aldrich (St Louis, MO, USA). All other chemicals were of reagent-grade and used without further purification.

\subsection{Preparation of Hydrogels}

PVA-PVP blended hydrogels were fabricated using a physical cross-linking method. First, each ingredient (PVA, PVP K-90, PVP K-30) was weighed, as indicated in Table 2. Then these ingredients were mixed in a beaker with the required amount of water. Subsequently, the beaker was sealed and heated for $25 \mathrm{~min}$ at $65-70{ }^{\circ} \mathrm{C}$ until a clear gel was obtained. To prevent the loss of water due to evaporation, the weight of the hydrogel was equalized with the initial water content and then the required amounts of PG and glycerol were added. Finally, DH was added and stirred for $5 \mathrm{~min}$. The prepared hydrogel formulation was centrifuged at $2852 \times g$ for 5 min at $25^{\circ} \mathrm{C}$ (Combi-514R; Hanil Science Industrial Co. Ltd., Gimpo, Korea) to remove air bubbles. After centrifugation, the prepared hydrogel was poured into a specially-designed mold, as shown in Figure 1, using a viscous liquid pouring pipette (Microman, Gilson ${ }^{\circledR}$ pipette; Gilson Inc., Middleton, WI, USA) to minimize errors and to maintain constant weights of all the hydrogels. Afterward, the hydrogels in molds were chilled at $4{ }^{\circ} \mathrm{C}$ for $24 \mathrm{~h}$ and then thawed at room temperature for $2 \mathrm{~h}$. Finally, the prepared hydrogels were stored in airtight Petri dishes until further use.

Table 2. Composition of prepared hydrogels $(\%, w / w)$.

\begin{tabular}{ccccc}
\hline Ingredients & 0\% PG-Hydrogel & 1\% PG-Hydrogel & $\mathbf{1 1 \%}$ PG-Hydrogel & 26\% PG-Hydrogel \\
\hline PVA & 7.5 & 7.5 & 7.5 & 7.5 \\
PVP K-90 & 8 & 8 & 8 & 8 \\
PVP K-30 & 2 & 2 & 2 & 2 \\
PG & 0 & 1 & 11 & 26 \\
Glycerol & 1 & 1 & 1 & 1 \\
DH & 11.7 & 11.7 & 11.7 & 11.7 \\
Water & 69.8 & 68.8 & 58.8 & 43.8 \\
\hline Total & 100 & 100 & 100 & 100 \\
\hline
\end{tabular}




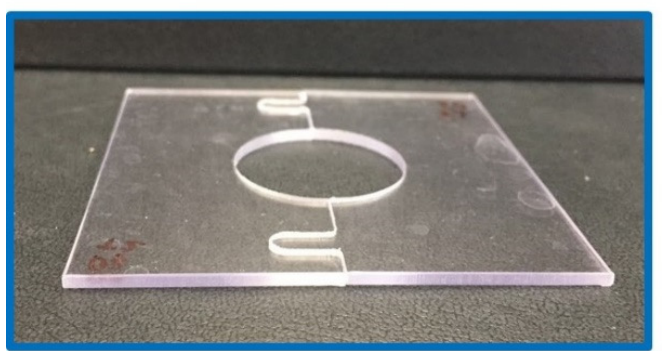

(A)

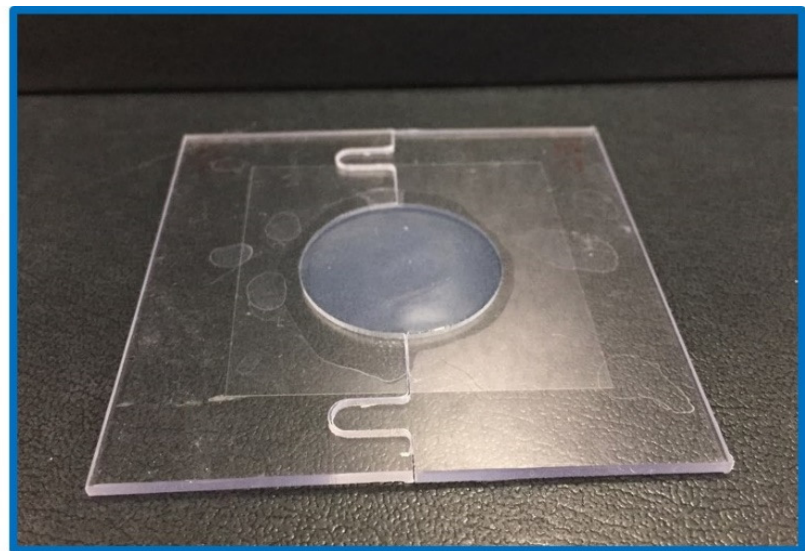

(B)

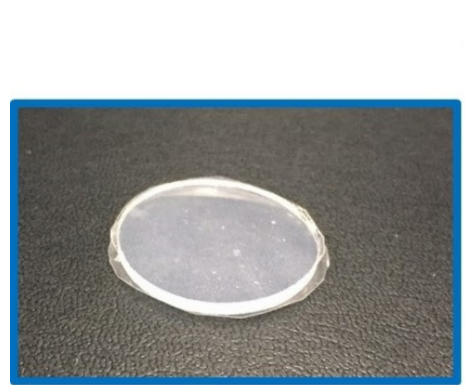

(C)
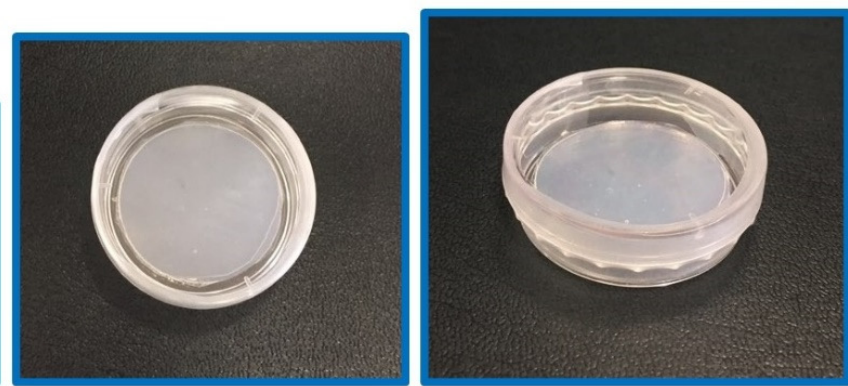

(D)

Figure 1. The specially designed mold for fabrication of circular hydrogels. A blank mold (A), a mold with an optimized hydrogel (26\% PG [propylene glycol]-hydrogel) (B), 26\% PG-hydrogel after its removal from the mold (C), 26\% PG-hydrogel in a Petri dish sealed with parafilm (D).

\subsection{Determination of Gel Fraction (\%)}

To measure the gel fraction, circular hydrogel samples $(2 \mathrm{~cm}$ diameter and $2 \mathrm{~mm}$ thickness) were prepared and then dried in an oven for $24 \mathrm{~h}$ at $50{ }^{\circ} \mathrm{C}\left(W_{o}\right)$. After $24 \mathrm{~h}$, dried hydrogel samples were immersed in $1000 \mathrm{~mL}$ distilled water for $24 \mathrm{~h}$ to reach equilibrium swelling and to remove the soluble parts. Subsequently, the samples were dried again in an oven at $50{ }^{\circ} \mathrm{C}\left(W_{e}\right)$ for $24 \mathrm{~h}$ [26]. The gel fraction was calculated as follows:

$$
\text { Gel fraction }(\%)=\frac{W_{e}}{W_{o}} \times 100
$$

\subsection{Determination of Swelling Ratio (\%)}

To measure water uptake capacity, circular hydrogel samples $(2 \mathrm{~cm}$ diameter and $2 \mathrm{~mm}$ thickness) were prepared and dried in an oven at $50^{\circ} \mathrm{C}$ for $24 \mathrm{~h}$. Then the weights of dried hydrogels $\left(W_{e}\right)$ were measured immediately. Subsequently, samples were immersed in distilled water at $37^{\circ} \mathrm{C}$ for $24 \mathrm{~h}$. Finally, the weight of the soaked hydrogel samples was measured $\left(W_{s}\right)$ [26]. The swelling ratio was calculated as follows:

$$
\text { Swelling ratio }(\%)=\frac{W_{s}-W_{e}}{W_{e}} \times 100
$$

\subsection{Weight Loss (\%)}

To measure the rate of water evaporation, circular hydrogel samples $(2 \mathrm{~cm}$ diameter and $2 \mathrm{~mm}$ thickness) were prepared, weighed $\left(W_{o}\right)$, and then stored in an oven at $37^{\circ} \mathrm{C}$. Subsequently, the weights 
of the samples were measured at different time points $\left(W_{t}\right)$ [29]. The percentage of weight loss was calculated as follows:

$$
\text { Weight loss }(\%)=\frac{W_{t}}{W_{o}} \times 100
$$

\subsection{Determination of the Mechanical Properties}

To measure the mechanical tensile strength and elongation at break, prepared hydrogel samples were cut into a specific "dog bone" shape, i.e., $6 \mathrm{~cm}$ long, $1.6 \mathrm{~cm}$ wide at the ends, and $0.8 \mathrm{~cm}$ wide in the middle, and then evaluated using a ball screw-driven motorized test frame (Mecmesin Multitest 2.5i, Mecmesin, Sterling, VA, USA) equipped with a $50 \mathrm{~N}$ load cell. Subsequently, the samples were clamped between tensile grips. The initial distance between the grips was $20 \mathrm{~mm}$, and the crosshead speed was $20 \mathrm{~mm} / \mathrm{min}$. The thickness of each hydrogel was also measured with a Vernier caliper. Tensile strength and breaking elongation at break were evaluated from stress-strain curves. Young's modulus was also calculated as the slope of the linear line of the stress-strain curve $[30,31]$.

\subsection{In Vitro Skin Permeation Study}

The skin preparation and in vitro skin permeation study were performed using our previous well-established method [32,33]. Briefly, the skin was obtained from male Sprague-Dawley rats (7-8 weeks old, 220-240 g). Rats were anesthetized using an intraperitoneal injection of urethane and the hair from the dorsum was removed with electric clippers. Subsequently, a depilatory was applied to detach remaining hair and then the skin was excised using surgical scissors. The underlying adipose tissue from the skin was carefully eliminated with scalpel and forceps and stored at $-20^{\circ} \mathrm{C}$ until further use.

The in vitro permeation study was performed using rat skin in a vertical static Franz diffusion cell with an effective diffusion area of $1.77 \mathrm{~cm}^{2}$. The skin tissue was fixed horizontally between the donor and receptor compartments. The hydrogel sample (equivalent to $37 \mathrm{mg} \mathrm{DH}$ ) was then placed above the skin, and the study was performed from the donor to the receptor compartment of the Franz diffusion cell filled with phosphate-buffered saline ( $\mathrm{pH}$ 7.4) with constant stirring using a magnetic stirrer at $600 \mathrm{rpm}$, maintained at $37^{\circ} \mathrm{C}$. A total of $0.5 \mathrm{~mL}$ of sample was withdrawn from the receptor compartment at pre-determined time points over $72 \mathrm{~h}$ and replaced immediately with an equal volume of phosphate-buffered saline ( $\mathrm{pH}$ 7.4) to maintain a constant volume. Each experiment was performed in triplicate.

The amount of permeated DH was determined by reverse-phase high-performance liquid chromatography (RP-HPLC) using a previously reported method [34]. Briefly, an Agilent 1200 infinity series LC system (Agilent Technologies Inc., Santa Clara, CA, USA) was used with an Agilent Eclipse XDB-C18 column $(5 \mu \mathrm{m}, 4.6 \times 250 \mathrm{~mm})$. The mobile phase was a mixture of methanol and $0.02 \mathrm{M}$ monobasic sodium phosphate (60:40) with a flow rate of $1 \mathrm{~mL} / \mathrm{min}$ and a UV detection wavelength of $268 \mathrm{~nm}$. Phosphate buffer was prepared by dissolving monobasic sodium phosphate in distilled water, followed by adding $10 \mathrm{~mL}$ of triethylamine and adjusting the $\mathrm{pH}$ to $2.7 \pm 0.5$ with phosphoric acid.

The steady-state flux $\left(J_{s}\right)$, permeability coefficient $\left(K_{p}\right)$, lag time, and enhancement ratio (ER) were calculated as described previously $[32,35]$. Briefly, $J_{s}$ was calculated as follows:

$$
J_{s}=\frac{Q_{r}}{A \cdot t}\left(\mathrm{mg} \cdot \mathrm{cm}^{-2} \cdot \mathrm{h}^{-1}\right)
$$

where $Q_{r}, A$, and $t$ are the total permeated $\mathrm{DH}(\mathrm{mg})$, diffusion area $\left(\mathrm{cm}^{2}\right)$, and the time of exposure (h), respectively.

Similarly, $K_{p}$ was calculated as follows:

$$
K_{p}=\frac{J_{s}}{C_{d}}\left(\mathrm{~cm} \cdot \mathrm{h}^{-1}\right)
$$


where $C_{d}$ is the initial concentration of $\mathrm{DH}\left(\mathrm{mg} \cdot \mathrm{cm}^{-3}\right)$. In addition, the lag time was calculated from the $x$-intercept of the linear regression line. Finally, ER was calculated by the ratio of the $K_{p}$ value of each hydrogel formulation to that of the control ( $0 \%$ PG-hydrogel).

\subsection{Animals}

Male, hairless, 6-week-old rats were obtained from Central Lab Animal Inc. (Seoul, Korea). Animals were maintained at $(23 \pm 2){ }^{\circ} \mathrm{C}$ under a $12 \mathrm{~h}$ light/dark cycle (lights on 07:00-19:00) and were provided with access to food and water ad libitum. All animal studies were approved by the Institutional Animal Care and Use Committee (IACUC: I-1903046, 14 March 2019) of the Research Institute of Dong-A ST.

\subsection{Pharmacokinetic Study in Hairless Rats}

To assess the pharmacokinetic profiles, hairless rats with an average weight of 450-500 g were randomly assigned to corresponding groups of five animals per group. The hairless rats were anesthetized with diethyl ester, patched, and dressed in adhesive stretch bandages for patch fixation. To evaluate dose effects, the optimized hydrogel (26\% PG-hydrogel) was prepared with two different proportions of $\mathrm{DH}(5.85 \%$ and $11.7 \%)$. DH hydrogel-patches were applied transdermally to the back skin of hairless rats. Blood $(250 \mu \mathrm{L})$ was collected from the tail vein at each indicated time point: $0,8,24,30,48$, and $72 \mathrm{~h}$. Similarly, DH $(1 \mathrm{mg} / \mathrm{kg})$ was administered intravenously, and the blood $(250 \mu \mathrm{L})$ was collected from the tail vein at each indicated time point: $0,0.25,0.5,1,2,4,6,8$, and $24 \mathrm{~h}$. These samples were kept at $-80^{\circ} \mathrm{C}$ until analysis.

\subsection{Analysis of Plasma Donepezil Hydrochloride (DH) Levels}

Plasma DH concentrations were analyzed using LC-MS/MS with a slight modification of a previously reported method [36,37]. Briefly, DH $(10 \mathrm{mg})$ was accurately weighed into a $20 \mathrm{~mL}$ volumetric vial and dissolved in methanol to prepare a working stock solution of $1000 \mu \mathrm{g} / \mathrm{mL}$. An aliquot $(100 \mu \mathrm{L})$ of working stock solution $(1000 \mu \mathrm{g} / \mathrm{mL})$ was transferred to a $1 \mathrm{~mL}$ E-tube and serially diluted with methanol to obtain working solutions ranging from 78 to $1000 \mathrm{ng} / \mathrm{mL}$. Depending on the calibration range and matrix (plasma) used, samples were prepared in the following manner. A suitable aliquot of samples or control matrix was accurately pipetted into a $2 \mathrm{~mL}$ tube and spiked with the internal standard solution (amantadine $250 \mathrm{ng} / \mathrm{mL} ; 300 \mu \mathrm{L}$ ). For calibration and quality control (QC) samples, a suitable volume of calibration and QC spiking solution was added. Standards were diluted to a final concentration ranging from 3.9 to $500 \mathrm{ng} / \mathrm{mL}$. All samples were analyzed using LC-MS/MS with an Agilent Technologies 1200 series HPLC system coupled to a 6430 Triple Quad LC/MS (Santa Clara, CA, USA). Chromatography was performed on a Union UK-C18 $3 \mu$ column $(50 \times 2.0 \mathrm{~mm}$; Portland, OR, USA). A sample aliquot of $5 \mu \mathrm{L}$ was injected onto the LC-MS/MS system with an auto-sampler followed by a needle wash using methanol. A gradient HPLC system (mobile ratio [A:B] = 25:75) was used with mobile phases (A) formic acid $(0.05 \%)$, and (B) methanol $(100 \%)$ at a total flow rate of $0.2 \mathrm{~mL} / \mathrm{min}$. Data were obtained using proprietary software from the instrument manufacturer. Peak areas and quantitative data were generated using MassHunter Workstation Software Quantitative Analysis Version B 04.00 (Agilent Technologies; Santa Clara, CA, USA). To compare the bioavailability of transdermal hydrogel-patches of $\mathrm{DH}$ with the bioavailability of $\mathrm{DH}$ following intravenous administration, absolute bioavailability $(\mathrm{F})$ was determined. $\mathrm{F}$ was calculated as follows:

$$
F=\frac{A U C_{\text {transdermal }}}{A U C_{I V}} \times \frac{\text { Dose }_{I V}}{\text { Dose }_{\text {transdermal }}}
$$




\subsection{Statistical Analysis}

The results are expressed as the mean \pm standard deviation. One-way analysis of variance followed by Tukey's multiple comparison test was used to determine statistically significant differences between the groups. Results of $p<0.05$ were considered statistically significant.

\section{Results and Discussion}

\subsection{Preparation and Characterization of Hydrogels}

Hydrogels are a cross-linked network of hydrophilic polymers. Hydrogels have received considerable attention among drug delivery scientists, medical doctors, biomaterials scientists, and tissue engineers for various biomedical applications [38]. The selection of polymer is crucial for the fabrication of hydrogels. We prepared various hydrogels with PVA and two different grades of PVP (K-90, K-30) using a physical cross-linking method. This method involves simple freeze-thawing steps. In this study, we incubated a PVA-PVP blended hydrogel for $24 \mathrm{~h}$ at $4{ }^{\circ} \mathrm{C}$ as a freezing step, followed by $2 \mathrm{~h}$ thawing at room temperature. This method overcomes toxicity issues and poor mechanical strength attributed to chemical and irradiation methods [28]. We used PG as a solubilizer and a chemical enhancer. We screened and prepared various hydrogels with PVA, PVP, and PG with DH at different percentages. We observed that 7.5\% PVA, 10\% PVP, and 26\% PG showed optimal morphological characteristics, i.e., transparency, elasticity, gel formation, adhesiveness, and mechanical properties. Further, we optimized the percentage of PVP K-90 and PVP K-30 to be $8 \%$ and 2\%, respectively, based on the adhesive properties, as we observed that PVP K-90 imparts higher adhesiveness to the hydrogel than PVP K-30. After 2-3 days, there was leakage of liquid from the hydrogel. Thus, to surmount this problem, $1 \%$ glycerol (a humectant) was added. Finally, the optimized hydrogel was $26 \%$ PG-hydrogel (Figure 1). The organoleptic property of optimized hydrogel is presented in Table 3.

Table 3. Characteristics of optimized hydrogels according to visual observation.

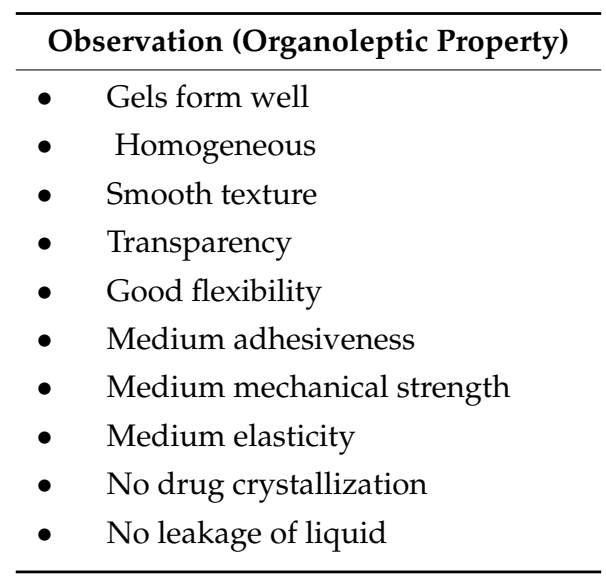

\subsubsection{Determination of the Gel Fraction (\%)}

To evaluate the effect of PG on cross-linking density and network formation, we measured gel fractions of various hydrogels with or without the drug. The influence of PG on the gel fraction (\%) is illustrated in Figure 2. This study revealed that the gel fraction (\%) increased as the concentration of PG decreased. In the absence of both the drug and PG and the presence of the drug (with drug and $0 \%$ PG), the gel fraction (\%) was increased to the maximum, and the value was $45.13 \pm 0.46$ and $41.33 \pm 0.14$, respectively. There was no significant difference in gel fraction (\%) with or without the drug. This finding confirmed that PVA-PVP was completely cross-linked and entangled. It has been reported that PVA and PVP are readily blended and cross-linked in their homogeneous mixture with water [24]. 


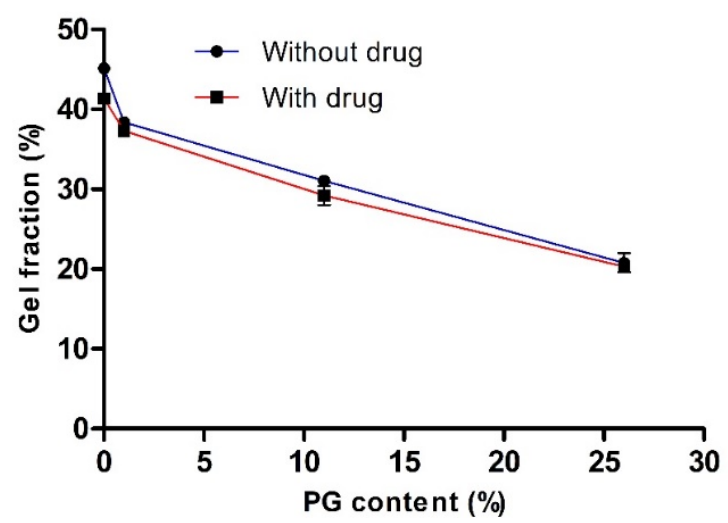

Figure 2. The effect of propylene glycol (PG) on the gel fraction (\%). Data are expressed as the mean \pm standard deviation $(n=4)$.

Nevertheless, the gel fraction (\%) was reduced with increased PG content and the value reached to $20.77 \pm 1.19$ and $20.29 \pm 0.12$ at $26 \%$ PG content, in the absence and presence of the drug, respectively. This finding may be because, in the presence of drug and PG, the cross-linking density may decrease, resulting in fewer entanglement reactions in the PVA-PVP hydrogel. Consequently, the cross-linking network chains are reduced by reducing physical interactions between the PVA and PVP network and, thus decreased the gelation process. These results were in accordance with the observation of previously obtained results $[26,39,40]$. Harthi et al. recently studied the gelation behavior of PVP hydrogels with different contents of polyethylene glycol (PEG) and found that the gel fraction (\%) was reduced as the concentration of PEG increased, while the gel fraction (\%) was maximum at $0 \%$ PEG content (PVP hydrogel without PEG content) [26]. In addition, the gelation behavior of PVA hydrogel was maximal, almost 90\%, in the absence of sodium alginate (SA) and decreased with the increasing content of SA, i.e., a $27 \%$ gel fraction at $30 \%$ SA [39].

\subsubsection{Determination of the Swelling Ratio (\%)}

To investigate the influence of PG on water engrossment capacity, we measured the swelling ratio (\%) of various hydrogels with or without the drug. The effect of PG on the swelling ratio (\%) is presented in Figure 3. This data clearly showed that the swelling ratio (\%) was increased with increasing concentration of PG. In the absence and presence of the drug (without drug and $26 \%$ PG; with drug and $26 \% \mathrm{PG}$ ), the swelling ratio (\%) was increased to the maximum and the value reached $289.32 \pm 5.36$ and $310.89 \pm 5.82$, respectively. There were no significant differences in the swelling ratio (\%) with or without the drug. This finding may be attributed to the PG content, as PG is a hydrophilic and hygroscopic molecule, which results in uptake of a large amount of water and does not take part in the cross-linking network. In general, cross-linking density has an inverse relationship with the swelling ratio [41]. However, in the absence and presence of drug (without drug and $0 \%$ PG; with drug and $0 \% \mathrm{PG})$, the swelling ratio (\%) was reduced with the decreased PG content, with values reaching $181.11 \pm 2.73$ and $191.11 \pm 2.36$ at $0 \%$ PG content, respectively, resulting in high cross-linking density of PVA-PVP blended hydrogels. Consequently, this network formation takes up small amounts of water.

These results were consistent with previously reported studies $[26,39,40]$. Kamoun et al. studied water uptake (\%) behavior of PVA hydrogels with and without drug and found that the uptake ratio (\%) with drug was maximized with a hydrogel containing $75 \%$ SA (4200\%), while at $0 \%$ SA content, the uptake ratio (\%) decreased to $1500 \%$. It has been reported that higher SA concentrations enhanced the wettability and hydrophilic nature of hydrogels, resulting in a higher swelling ratio [40]. In another study, the swelling ratio of a PVP hydrogel with 3\% PEG revealed higher swelling capacity than pure PVP hydrogel ( $0 \%$ PEG). The authors attributed this finding to the PEG content, as PEG is hydrophilic and drives swelling. This study concluded that with increased PEG content, the swelling ratio of PVP hydrogels increased gradually [26]. 


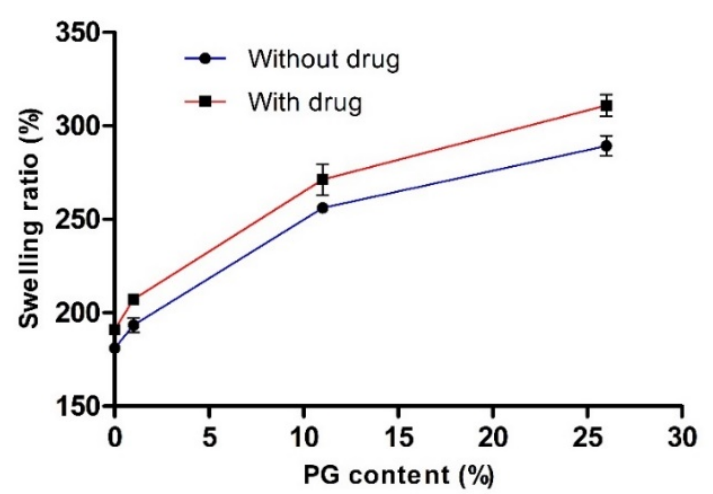

Figure 3. Effect of propylene glycol (PG) on the swelling ratio (\%). Data are expressed as the mean \pm standard deviation $(n=4)$.

\subsubsection{Determination of Weight Loss (\%)}

To evaluate the effect of PG on the water evaporation rate, we measured the weight loss (\%) of various hydrogels. The influence of PG on weight loss (\%) is illustrated at different time intervals in Figure 4 . This figure shows that the water loss from hydrogels increased gradually with time. It was found that the remaining weight (\%) of the control hydrogel, 1\% PG-hydrogel, 11\% PG-hydrogel, and $26 \%$ PG-hydrogel for the first $2 \mathrm{~h}$ were $66.23 \pm 0.67,67.64 \pm 1.12,70.84 \pm 3.80$, and $79.62 \pm 2.57$, respectively. As time passed, water loss gradually increased, and at certain times, it remained constant. After $8 \mathrm{~h}$, the remaining weight of the control hydrogel, the 1\% PG-hydrogel, and the 11\% PG-hydrogel were nearly identical, with an approximate water loss of $50 \%$. However, the remaining weight $(\%)$ of the $26 \%$ PG-hydrogel was $60.60 \pm 3.23$, i.e., the water loss was $40 \%$. It was observed that there was a slower loss of water from the hydrogel containing PG, and the loss of water was dependent upon PG content. This decreased loss is because PG is hydrophilic and hygroscopic. Finally, after $12 \mathrm{~h}$ and $24 \mathrm{~h}$, the remaining weight (\%) of the optimized hydrogel (26\% PG-hydrogel) was $56.23 \pm 2.47$ and $49.47 \pm 1.18$, respectively. The loss of water from marketed dressings such as Geliperm ${ }^{\circledR}$ (Geistlich Ltd., Wolhusen, Switzerland) is approximately $50 \%$ after $12 \mathrm{~h}$ [42]. Thus, our optimized hydrogel (26\% PG-hydrogel) showed similar behavior, i.e., after $12 \mathrm{~h}$ and $24 \mathrm{~h}$, the loss of water from the optimized hydrogel was approximately $44 \%$ and $51 \%$, respectively. These results were also compatible with previously reported results by Balakrishnan et al. [29].

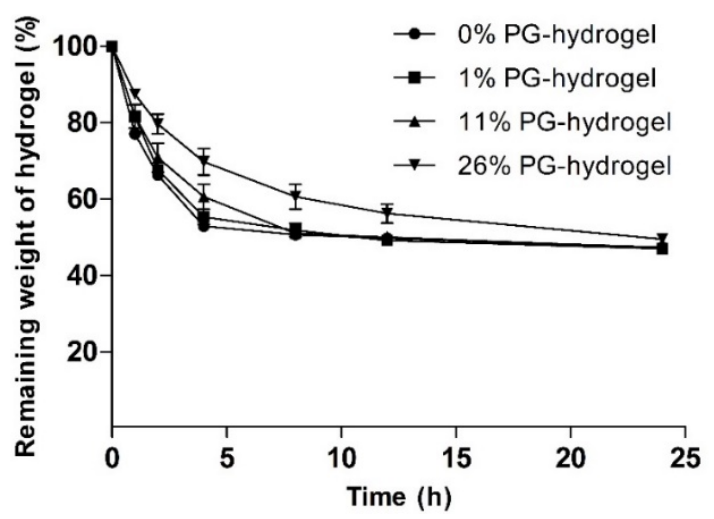

Figure 4. Effect of propylene glycol (PG) on weight loss (\%). Data are expressed as the mean \pm standard deviation $(n=3)$.

\subsubsection{Mechanical Properties Determinations}

To evaluate the effect of PG on the mechanical properties of $0 \%$ PG-hydrogel and 26\% PG-hydrogel samples, their tensile strength, elongation at break, and Young's modulus were measured. In the absence and presence of the drug, the maximum strength, elongation at break, and Young's modulus of 
the hydrogel decreased with increasing PG contents. However, the elongation at break of both hydrogels containing the drug remained similar to increasing content of PG. Overall, the results revealed that PG affected the cross-linking density of the hydrogels and weakened the breaking elongation, resulting in decreased mechanical strength as well as Young's modulus (Figure 5). These results are consistent with previously published reports $[40,43]$. The maximum tensile strength and elongation at break were sharply increased with the PVA hydrogel (0\% SA) and decreased with increasing concentration of SA [39].

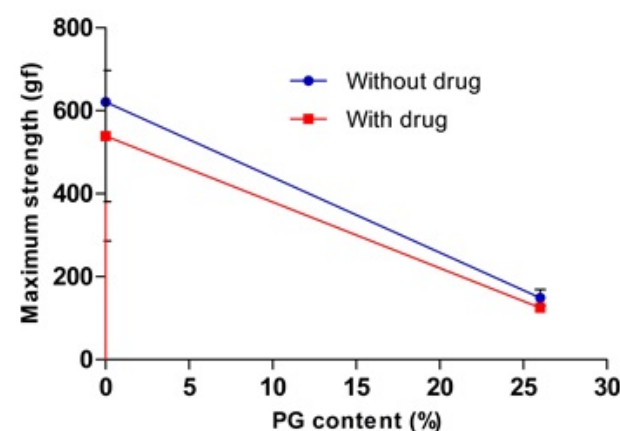

(A)

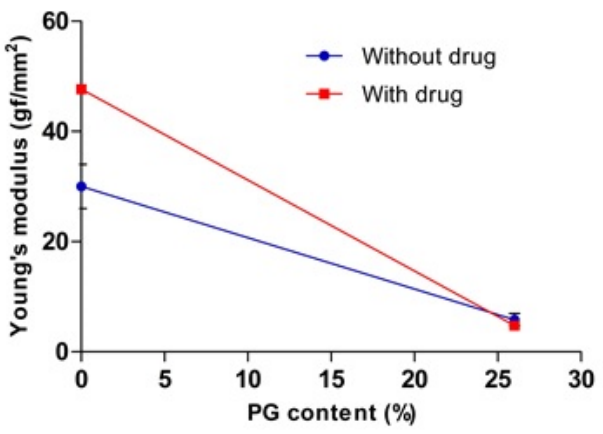

(C)

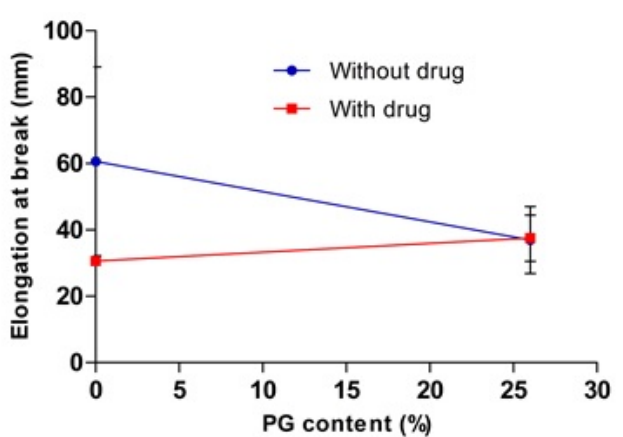

(B)

Figure 5. Effect of propylene glycol (PG) on the maximum strength (A), elongation at break (B), and Young's modulus $(\mathbf{C})$. Data are expressed as the mean \pm standard deviation $(n=3)$.

\subsection{In Vitro Skin Permeation Study}

To evaluate whether PG affected the permeation profiles of $\mathrm{DH}$, we performed an in vitro skin permeation study with various hydrogels for $72 \mathrm{~h}$. The cumulative permeation profiles of DH-loaded hydrogels through rat skin are illustrated as a function of time (Figure 6). The cumulative amount permeated by the $26 \%$ PG-hydrogel was approximately 13 times greater than that of the control hydrogel, 4.5 times greater than that of the 1\% PG-hydrogel, and twice greater than that permeated by the $11 \%$ PG-hydrogel. Alike to Corplex ${ }^{\mathrm{TM}}$ Donepezil (investigational transdermal formulation), the total amount permeated by the $26 \%$ PG-hydrogel at $24 \mathrm{~h}$ was $4.69 \mathrm{mg}$, approximately similar, aiming to deliver $5 \mathrm{mg}$ per day over the period of $72 \mathrm{~h}$ [44]. The skin permeation parameters $J_{s}, K_{p}$, and ER were calculated and are summarized in Table 4 . The rise of these parameters was observed in the order of 26\% PG-hydrogel > 11\% PG-hydrogel > 1\% PG-hydrogel > control ( $0 \%$ PG-hydrogel). The $26 \%$ PG-hydrogel revealed the highest $J_{s}$ and $K_{p}$, which were significantly greater than those of other hydrogel formulations (*** $p<0.001$ vs. control; \#\#\# $p<0.001$ vs. $1 \%$ PG-hydrogel and $\$ \$ \$ p<0.001$ vs. $11 \%$ PG-hydrogel). Similarly, the $J_{s}$ and $K_{p}$, for $11 \%$ PG-hydrogel were also significantly higher than the control hydrogel (*** $p<0.001$ ) and the 1\% PG-hydrogel (\#\#\# $p<0.001$ ). In addition, the $J_{s}$ and $K_{p}$ for the 1\% PG-hydrogel were also significantly greater than that of the control hydrogel $\left({ }^{*} p<0.05\right)$. The lag time of DH permeation was reduced 5.97 times by $26 \%$ PG-hydrogel compared to the control 
hydrogel. The 26\% PG-hydrogel had a higher ER (12.9-fold) than the control hydrogel. In addition, the 11\% PG-hydrogel and 1\% PG-hydrogel also exhibited ER values 6.30-fold and 2.85-fold higher than that of the control, respectively.

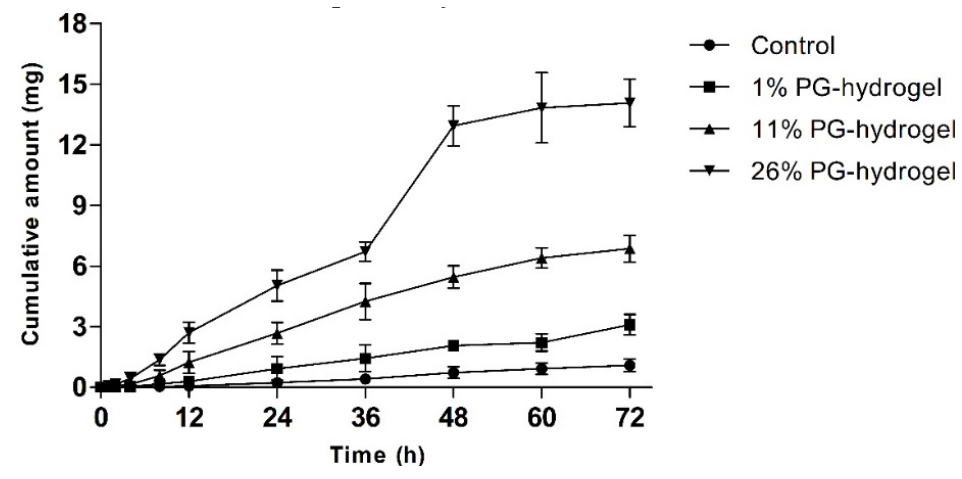

Figure 6. In vitro rat skin permeation by donepezil hydrochloride (DH). The effect of propylene glycol (PG) on transdermal delivery of DH. Error bars represent standard deviation $(n=3)$.

Table 4. Skin permeation parameters for different hydrogel formulations. Data are expressed as the mean \pm standard deviation $(n=3) .{ }^{*} p<0.05$ vs. control, ${ }^{* * *} p<0.001$ vs. control; \#\# $p<0.01$ vs. $1 \%$ PG [propylene glycol]-hydrogel, \#\#\# $p<0.001$ vs. 1\% PG-hydrogel; $\$ \$ p<0.001$ vs. 11\% PG-hydrogel.

\begin{tabular}{|c|c|c|c|c|}
\hline Hydrogel & $J_{s}\left(\mathrm{mg} \cdot \mathrm{cm}^{-2} \cdot \mathrm{h}^{-1}\right)$ & $K_{p}(\mathrm{~cm} / \mathrm{h}) \times 10^{-4}$ & Lag Time (h) & $\begin{array}{c}\text { Enhancement } \\
\text { Ratio (ER) }\end{array}$ \\
\hline Control & $0.009 \pm 0.002$ & $2.32 \pm 0.65$ & $3.76 \pm 0.66$ & 1.00 \\
\hline 1\% PG-hydrogel & $0.024 \pm 0.004$ * & $6.60 \pm 1.08^{*}$ & $2.10 \pm 3.05$ & 2.85 \\
\hline 11\% PG-hydrogel & $0.054 \pm 0.005^{* * *}$ & $14.62 \pm 1.40^{* * *}$,\#\# & $0.23 \pm 2.67$ & 6.30 \\
\hline $26 \%$ PG-hydrogel & $0.110 \pm 0.009^{* * *}, \# \# \#, \$ \$$ & $29.95 \pm 2.49^{* * *}, \# \# \#, \$ \$$ & $0.63 \pm 1.04$ & 12.90 \\
\hline
\end{tabular}

The permeation of the drug across the skin is greatly influenced by the polymer and the solvent used in the hydrogel formulation. The cross-linked network of a hydrogel is enhanced with the addition of PVA and PVP (K-90, K-30), and consequently decreases the elasticity and flexibility of hydrogels. However, the cross-linking density and network formation diminished with the incorporation of PG into the hydrogel, and thus, the elasticity and flexibility will be enhanced [26]. This behavior might be one crucial factor in increasing the skin permeation of DH. PG is a hygroscopic as well as a hydrophilic molecule [17]. It engrosses the water molecules from the receiver medium. This study revealed that $26 \%$ PG-containing hydrogel had the highest skin permeation of DH compared to the other hydrogels, and the hydrogels followed the concentration-dependent trend of PG, i.e., permeated amounts increased with increasing concentrations of PG. This finding is because PG acts as a solubilizing cosolvent, a permeation enhancer and has the potential to solvate the keratin of the stratum corneum [15]. Consequently, PG penetrates the skin tissues and occupies the hydrogen bonding sites leading to intracellular transport of drug and enhanced skin permeation [45]. These results were in agreement with previous reports $[46,47]$. PG has the potential to intercalate into the polar head groups of the lipid bilayer due to its hydrophilic nature and significantly enhances the mean interfacial area per lipid [48]. Moreover, skin partitioning and the barrier function of the skin are decreased by enhanced trans-epidermal water loss and kallikrein 7 (KLK 7) protease activity, which might account for the enhancement of skin permeation $[49,50]$. Yamane et al. reported that the flux and permeability coefficient of 5-fluorouracil was increased with the addition of terpenes to the PG system. The activity of terpenes was dependent upon the PG concentration, and the flux was maximized with a formulation containing 80\% PG, which demonstrates the concentration-dependent relationship between flux and PG content. It was concluded that the drug partitioning and lipid disruption are the main mechanisms, resulting in enhanced skin permeation across human epidermis [46]. 
In another study performed by Lee et al., PG acted as an enhancer and increased the partitioning of PG into the stratum corneum, leading to the maximum flux of acetaminophen with $30 \%$ PG content, compared with a 10\% PG formulation, which in turn was higher than a $0 \%$ PG-containing formulation [47]. Furthermore, the in vitro percutaneous permeation of loperamide hydrochloride and PG in a formulation containing $15 \%$ and $40 \%$ PG was tested, and low amounts of drug and substantial amounts of PG were permeated; this confirmed time-dependent penetration of the drug and suggested that the mechanism of permeability involved depletion of PG on the skin surface. In addition, there was a dose-dependent correlation between PG content and permeated drug [51]. Recently, a monolithic transdermal patch system incorporated with nanostructured lipid carriers (NLCs) was developed to co-deliver olanzapine and simvastatin, and screened with various chemical enhancers such as PG, Transcutol ${ }^{\circledR}$, menthol, or limonene to determine their skin permeation profiles. Among these, PG was the best enhancer for olanzapine and simvastatin with an ER of 8.05 and 12.89, respectively, when compared with the control (Combo-NLC patch without enhancer) [52]. Moreover, a molecular dynamics simulation study was performed to elucidate the mechanism and found that PG was intercalated on the hydrophilic region of the phospholipid bilayers and increased the lateral diffusion of phospholipid molecules, suggesting pronounced enhancement profiles due to increased fluidity by disruption of highly ordered lipid lamellae [52]. In addition, PG promoted the flux of metronidazole, triprolidine base, cinnamaldehyde, and ibuprofen [16].

\subsection{Pharmacokinetic Profiles of the DH Hydrogel-Patch in Hairless Rats}

To evaluate the feasibility of optimized hydrogel formulations, in vivo pharmacokinetic studies were performed in hairless rats after the transdermal application of DH hydrogel-patches. The plasma concentration-time profiles of $\mathrm{DH}$ following intravenous and transdermal administration are illustrated in Figure 7A,B. The pharmacokinetic parameters such as the time to reach the maximum concentration $\left(\mathrm{T}_{\max }\right)$, the maximum (or peak) plasma concentration $\left(\mathrm{C}_{\max }\right)$ and the area under the curve (AUC) were calculated and are summarized in Table 5 . An intravenous bolus of $1 \mathrm{mg} / \mathrm{kg}$ was administered to hairless rats (Figure 7A), and the $C_{\max }$ and AUC were $93.4 \pm 18.9 \mathrm{ng} / \mathrm{mL}$ and $133.4 \pm 23.5 \mathrm{ng} \cdot \mathrm{h} / \mathrm{mL}$, respectively. In addition, the $\mathrm{C}_{\max }$ for $\mathrm{DH}$ was achieved at $8 \mathrm{~h}$ and other parameters were dose-dependent after the application of transdermal DH hydrogel-patches. The $\mathrm{C}_{\max }$ of the $11.7 \% \mathrm{DH}$ hydrogel-patch and the $5.85 \% \mathrm{DH}$ hydrogel-patch were $255.4 \pm 95.3 \mathrm{ng} / \mathrm{mL}$ and $115.2 \pm 41.3 \mathrm{ng} / \mathrm{mL}$, respectively. Similarly, the AUC of the $11.7 \%$ DH hydrogel-patch was nearly 2 times higher than that of the $5.85 \%$ DH hydrogel-patch, suggesting dose-dependent characteristics of hydrogel-mediated permeation. The comparison of transdermal administration with that of intravenous administration of the same drug is indicated by F. The F of the $11.7 \%$ DH hydrogel-patch and the $5.85 \% \mathrm{DH}$ hydrogel-patch was determined using dose-normalized AUC values following oral and intravenous administration, indicating $\mathrm{F}$ itself depends on the dose and the rate of absorption. Thus, the $11.7 \% \mathrm{DH}$ hydrogel-patch and the $5.85 \%$ DH hydrogel-patch had similar normalized value of $\mathrm{F}$.

Table 5. Donepezil hydrochloride (DH) pharmacokinetic parameters after intravenous (IV) injection or application of DH hydrogel-patches to hairless rats. Data are expressed as the mean \pm standard deviation $(n=5)$.

\begin{tabular}{cccc}
\hline Parameter & IV & \multicolumn{2}{c}{ DH Hydrogel-Patch } \\
\hline Dose & $1 \mathrm{mg} / \mathrm{kg}$ & $5.85 \%$ & $11.7 \%$ \\
$\mathrm{~T}_{\max }(\mathrm{h})$ & - & $8.0 \pm 0.0$ & $8.0 \pm 0.0$ \\
$\mathrm{C}_{\max }(\mathrm{ng} / \mathrm{mL})$ & $93.4 \pm 18.9$ & $115.2 \pm 41.3$ & $255.4 \pm 95.3$ \\
$\mathrm{AUC}_{0 \rightarrow \text { last }}(\mathrm{ng} \cdot \mathrm{h} / \mathrm{mL})$ & $133.4 \pm 23.5$ & $1815.1 \pm 631.9$ & $3420.3 \pm 1087.6$ \\
$\mathrm{~F}$ & - & 0.35 & 0.33 \\
\hline
\end{tabular}




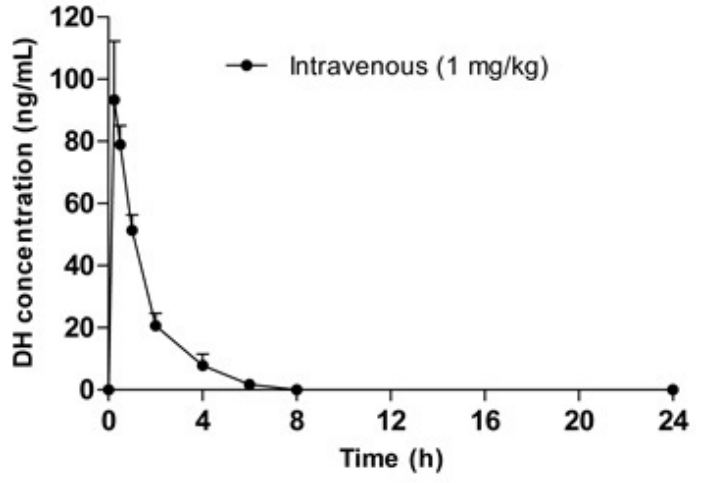

(A)

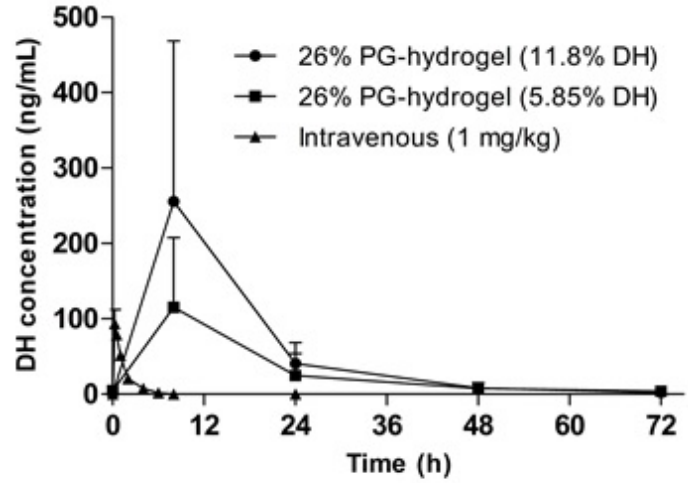

(B)

Figure 7. Pharmacokinetic profiles of donepezil hydrochloride (DH) in hairless rats. Intravenous injection of DH $(1 \mathrm{mg} / \mathrm{kg})(\mathrm{A})$ and the plasma concentration-time curve for $72 \mathrm{~h}$ after the application of DH hydrogel-patches $(\mathbf{B})$. Data are expressed as the mean \pm standard deviation $(n=5)$.

\section{Conclusions}

PVA-PVP blended hydrogels were developed using a physical cross-linking method. The entanglement of PG within the cross-linked network of PVA-PVP blended hydrogels notably influence its physicochemical properties such as gel fraction (\%), swelling ratio (\%), weight loss (\%), mechanical strength, breaking elongation, Young's modulus, and skin permeation, as well as in vivo pharmacokinetics profiles of DH. In summary, the application of this optimized hydrogel demonstrated a feasible alternative for transdermal delivery of $\mathrm{DH}$, and thus, hydrogel-based TDDS could be a promising platform for delivery of $\mathrm{DH}$ and for the effective treatment of $\mathrm{AD}$.

Author Contributions: Conceptualization, S.B., C.Y.S., S.W.J., and S.L.; methodology, S.B, C.Y.S., S.W.J., and S.L.; software, S.B., C.Y.S., and S.W.J; validation, S.B., C.Y.S., S.M.H, S.W.J., and S.L.; formal analysis, S.B. and C.Y.S.; investigation, S.B., C.Y.S., and S.M.H.; resources, S.W.J. and S.L.; data curation, S.B. and C.Y.S.; writing-original draft preparation, S.B.; writing-review and editing, S.B., S.W.J., and S.L.; visualization, S.B.; supervision, S.W.J. and S.L.; funding acquisition, S.W.J. and S.L. All authors have read and agreed to the published version of the manuscript.

Funding: The Senior-friendly Product R\&D program supported this research through the Korea Health Industry Development Institute, funded by the Ministry of Health \& Welfare, Republic of Korea (grant number: HI16C1960). This research was also supported by the Basic Science Research Program through the National Research Foundation of Korea (NRF-2016R1A6A1A03011325) funded by the Ministry of Education.

Acknowledgments: We would like to extend our deepest gratitude to the Research Institute of Dong-A ST Co. Ltd., Yongin 17073, Korea.

Conflicts of Interest: The authors declare no conflict of interest. Shin CY, Hyun SM, and Jang SW are employees of the Research Institute of Dong-A ST Co. Ltd. Keimyung university performed formulation design and characterization of hydrogels and Dong-A ST performed characterization of hydrogels, pharmacokinetic studies and analysis of blood samples.

\section{References}

1. Choi, J.; Choi, M.-K.; Chong, S.; Chung, S.-J.; Shim, C.-K.; Kim, D.-D. Effect of fatty acids on the transdermal delivery of donepezil: In vitro and in vivo evaluation. Int. J. Pharm. 2012, 422, 83-90. [CrossRef] [PubMed]

2. Alzheimer's Disease International. World Alzheimer Report 2015. Available online: https://www.alz.co.uk/ research/statistics (accessed on 29 January 2020).

3. Association, A.S. 2019 Alzheimer's disease facts and figures. Alzheimer's Dement. 2019, 15, 321-387. [CrossRef]

4. Bartus, R.T.; Dean, R.r.; Beer, B.; Lippa, A.S. The cholinergic hypothesis of geriatric memory dysfunction. Science 1982, 217, 408-414. [CrossRef]

5. Tariot, P.; Salloway, S.; Yardley, J.; Mackell, J.; Moline, M. Long-term safety and tolerability of donepezil $23 \mathrm{mg}$ in patients with moderate to severe Alzheimer's disease. BMC Res. Notes 2012, 5, 283. [CrossRef] 
6. Subedi, R.K.; Ryoo, J.-P.; Moon, C.; Chun, M.-K.; Choi, H.-K. Formulation and in vitro evaluation of transdermal drug delivery system for donepezil. J. Pharm. Investig. 2012, 42, 1-7. [CrossRef]

7. Kim, J.-Y.; Han, M.-R.; Kim, Y.-H.; Shin, S.-W.; Nam, S.-Y.; Park, J.-H. Tip-loaded dissolving microneedles for transdermal delivery of donepezil hydrochloride for treatment of Alzheimer's disease. Eur. J. Pharm. Biopharm. 2016, 105, 148-155. [CrossRef]

8. Saluja, S.; Kasha, P.C.; Paturi, J.; Anderson, C.; Morris, R.; Banga, A.K. A novel electronic skin patch for delivery and pharmacokinetic evaluation of donepezil following transdermal iontophoresis. Int. J. Pharm. 2013, 453, 395-399. [CrossRef]

9. Galipoğlu, M.; Erdal, M.S.; Güngör, S. Biopolymer-based transdermal films of donepezil as an alternative delivery approach in Alzheimer's disease treatment. AAPS PharmSciTech 2015, 16, 284-292. [CrossRef] [PubMed]

10. Sozio, P.; Cerasa, L.S.; Marinelli, L.; Di Stefano, A. Transdermal donepezil on the treatment of Alzheimer's disease. Neuropsychiatr. Dis. Treat. 2012, 8, 361.

11. Hajjar, B.; Zier, K.-I.; Khalid, N.; Azarmi, S.; Löbenberg, R. Evaluation of a microemulsion-based gel formulation for topical drug delivery of diclofenac sodium. J. Pharm. Investig. 2018, 48, 351-362. [CrossRef]

12. Rastogi, V.; Yadav, P.; Verma, N.; Verma, A. Preparation and characterization of transdermal mediated microemulsion delivery of T4 bacteriophages against E. coli bacteria: A novel anti-microbial approach. J. Pharm. Investig. 2018, 48, 393-407. [CrossRef]

13. Bashyal, S.; Lee, S. Delivery of biopharmaceuticals using combination of liposome and iontophoresis: A review. J. Pharm. Investig. 2015, 45, 611-624. [CrossRef]

14. Lee, A.-R.C. Microneedle-mediated delivery of cosmeceutically relevant nucleoside and peptides in human skin: Challenges and strategies for dermal delivery. J. Pharm. Investig. 2019, 49, 587-601.

15. Karande, P.; Mitragotri, S. Enhancement of transdermal drug delivery via synergistic action of chemicals. Biochim. Biophys. Acta 2009, 1788, 2362-2373. [CrossRef]

16. Lane, M.E. Skin penetration enhancers. Int. J. Pharm. 2013, 447, 12-21. [CrossRef]

17. Rowe, R.C.; Sheskey, P.J.; Quinn, M.E. Handbook of Pharmaceutical Excipients, 6th ed.; Pharmaceutical Press: London, UK, 2009; pp. 592-593.

18. Trommer, H.; Neubert, R. Overcoming the stratum corneum: The modulation of skin penetration. Skin Pharmacol. Physiol. 2006, 19, 106-121. [CrossRef]

19. Dai, Y.N.; Li, P.; Zhang, J.P.; Wang, A.Q.; Wei, Q. A novel pH sensitive N-succinyl chitosan/alginate hydrogel bead for nifedipine delivery. Biopharm. Drug Dispos. 2008, 29, 173-184. [CrossRef]

20. Wona, G.; Janik, H. Review: Synthetic polymer hydrogels forbiomedical application. Chem. Chem. Technol. 2010, 4, 297-304.

21. Rac, V.; Lević, S.; Balanč, B.; Graells, B.O.; Bijelić, G. PVA Cryogel as model hydrogel for iontophoretic transdermal drug delivery investigations. Comparison with PAA/PVA and PAA/PVP interpenetrating networks. Colloids Surf. B Biointerfaces 2019, 180, 441-448. [CrossRef]

22. Hamidi, M.; Azadi, A.; Rafiei, P. Hydrogel nanoparticles in drug delivery. Adv. Drug Deliv. Rev. 2008, 60, 1638-1649. [CrossRef]

23. Mansour, L.M.; Taj, B.M.; Mokhtari, M. Synthesis and swelling characterization of cross-linked PVP/PVA hydrogels. Iran. Polym. J. 2005, 14, 1022-1030.

24. Nho, Y.-C.; Lim, Y.-M.; Gwon, H.-J.; Choi, E.-K. Preparation and characterization of $\mathrm{PVA} / \mathrm{PVP} /$ glycerin/antibacterial agent hydrogels using $\gamma$-irradiation followed by freeze-thawing. Korean J. Chem. Eng. 2009, 26, 1675-1678. [CrossRef]

25. Thomas, J.; Lowman, A.; Marcolongo, M. Novel associated hydrogels for nucleus pulposus replacement. J. Biomed. Mater. Res. A 2003, 67, 1329-1337. [CrossRef] [PubMed]

26. Harthi, S.A.; Alavi, S.E.; Radwan, M.A.; El Khatib, M.M.; AlSarra, I.A. Nasal delivery of donepezil HCl-loaded hydrogels for the treatment of Alzheimer's disease. Sci. Rep. 2019, 9, 1-20. [CrossRef] [PubMed]

27. Razzak, M.T.; Darwis, D. Irradiation of polyvinyl alcohol and polyvinyl pyrrolidone blended hydrogel for wound dressing. Radiat. Phys. Chem. 2001, 62, 107-113. [CrossRef]

28. Hassan, C.M.; Peppas, N.A. Structure and applications of poly (vinyl alcohol) hydrogels produced by conventional crosslinking or by freezing/thawing methods. In Biopolymers PVA Hydrogels, Anionic Polymerisation Nanocomposites; Springer: New York, NY, USA, 2000; pp. 37-65. 
29. Balakrishnan, B.; Mohanty, M.; Umashankar, P.; Jayakrishnan, A. Evaluation of an in situ forming hydrogel wound dressing based on oxidized alginate and gelatin. Biomaterials 2005, 26, 6335-6342. [CrossRef]

30. Soazo, M.; Báez, G.; Barboza, A.; Busti, P.A.; Rubiolo, A.; Verdini, R.; Delorenzi, N. Heat treatment of calcium alginate films obtained by ultrasonic atomizing: Physicochemical characterization. Food Hydrocoll. 2015, 51, 193-199. [CrossRef]

31. Travan, A.; Scognamiglio, F.; Borgogna, M.; Marsich, E.; Donati, I.; Tarusha, L.; Grassi, M.; Paoletti, S. Hyaluronan delivery by polymer demixing in polysaccharide-based hydrogels and membranes for biomedical applications. Carbohydr. Polym. 2016, 150, 408-418. [CrossRef]

32. Noh, G.; Keum, T.; Seo, J.-E.; Bashyal, S.; Eum, N.-S.; Kweon, M.; Lee, S.; Sohn, D.; Lee, S. Iontophoretic transdermal delivery of Human Growth Hormone $(\mathrm{hGH})$ and the combination effect of a new type Microneedle, Tappy Tok Tok ${ }^{\circledR}$. Pharmaceutics 2018, 10, 153. [CrossRef]

33. Jeon, H.S.; Seo, J.E.; Kim, M.S.; Kang, M.H.; Oh, D.H.; Jeon, S.O.; Jeong, S.H.; Choi, Y.W.; Lee, S. A retinyl palmitate-loaded solid lipid nanoparticle system: Effect of surface modification with dicetyl phosphate on skin permeation in vitro and anti-wrinkle effect in vivo. Int. J. Pharm. 2013, 452, 311-320. [CrossRef]

34. Pappa, H.; Farrú, R.; Vilanova, P.O.; Palacios, M.; Pizzorno, M.a.T. A new HPLC method to determine Donepezil hydrochloride in tablets. J. Pharm. Biomed. Anal. 2002, 27, 177-182. [CrossRef]

35. Bashyal, S.; Seo, J.-E.; Keum, T.; Noh, G.; Choi, Y.W.; Lee, S. Facilitated permeation of insulin across TR146 cells by cholic acid derivatives-modified elastic bilosomes. Int. J. Nanomed. 2018, 13, 5173. [CrossRef] [PubMed]

36. Geerts, H.; Guillaumat, P.-O.; Grantham, C.; Bode, W.; Anciaux, K.; Sachak, S. Brain levels and acetylcholinesterase inhibition with galantamine and donepezil in rats, mice, and rabbits. Brain Res. 2005, 1033, 186-193. [CrossRef] [PubMed]

37. Bhateria, M.; Ramakrishna, R.; Pakala, D.B.; Bhatta, R.S. Development of an LC-MS/MS method for simultaneous determination of memantine and donepezil in rat plasma and its application to pharmacokinetic study. J. Chromatogr. B Anal. Technol. Biomed. Life Sci. 2015, 1001, 131-139. [CrossRef] [PubMed]

38. Gupta, S.; Webster, T.J.; Sinha, A. Evolution of PVA gels prepared without crosslinking agents as a cell adhesive surface. J. Mater. Sci. Mater. Med. 2011, 22, 1763-1772. [CrossRef]

39. Kim, J.O.; Park, J.K.; Kim, J.H.; Jin, S.G.; Yong, C.S.; Li, D.X.; Choi, J.Y.; Woo, J.S.; Yoo, B.K.; Lyoo, W.S. Development of polyvinyl alcohol-sodium alginate gel-matrix-based wound dressing system containing nitrofurazone. Int. J. Pharm. 2008, 359, 79-86. [CrossRef]

40. Kamoun, E.A.; Kenawy, E.-R.S.; Tamer, T.M.; El-Meligy, M.A.; Eldin, M.S.M. Poly (vinyl alcohol)-alginate physically crosslinked hydrogel membranes for wound dressing applications: Characterization and bio-evaluation. Arab. J. Chem. 2015, 8, 38-47. [CrossRef]

41. Mi, F.L.; Shyu, S.S.; Lee, S.T.; Wong, T.B. Kinetic study of chitosan-tripolyphosphate complex reaction and acid-resistive properties of the chitosan-tripolyphosphate gel beads prepared by in-liquid curing method. J. Polym. Sci. Pol. Phys. 1999, 37, 1551-1564. [CrossRef]

42. Kickhöfen, B.; Wokalek, H.; Scheel, D.; Ruh, H. Chemical and physical properties of a hydrogel wound dressing. Biomaterials 1986, 7, 67-72. [CrossRef]

43. Hwang, M.-R.; Kim, J.O.; Lee, J.H.; Kim, Y.I.; Kim, J.H.; Chang, S.W.; Jin, S.G.; Kim, J.A.; Lyoo, W.S.; Han, S.S. Gentamicin-loaded wound dressing with polyvinyl alcohol/dextran hydrogel: Gel characterization and in vivo healing evaluation. AAPS PharmsciTech 2010, 11, 1092-1103. [CrossRef]

44. Corium International Inc. Lead Investigational Product. Available online: http://www.coriumintl.com (accessed on 9 March 2020).

45. Sinha, V.; Kaur, M.P. Permeation enhancers for transdermal drug delivery. Drug Dev. Ind. Pharm. 2000, 26, 1131-1140. [CrossRef] [PubMed]

46. Yamane, M.; Williams, A.; Barry, B. Terpene penetration enhancers in propylene glycol/water co-solvent systems: Effectiveness and mechanism of action. J. Pharm. Pharmacol. 1995, 47,978-989. [CrossRef] [PubMed]

47. Lee, S.Y.; Jeong, N.Y.; Oh, S.Y. Modulation of electroosmosis and flux through skin: Effect of propylene glycol. Arch. Pharm. Res. 2014, 37, 484-493. [CrossRef] [PubMed]

48. Bouwstra, J.; De Vries, M.; Gooris, G.; Bras, W.; Brussee, J.; Ponec, M. Thermodynamic and structural aspects of the skin barrier. J. Control Release 1991, 15, 209-219. [CrossRef]

49. Haque, T.; Talukder, M.M.U. Chemical enhancer: A simplistic way to modulate barrier function of the stratum corneum. Adv. Pharm. Bull. 2018, 8, 169. [CrossRef] 
50. Mohammed, D.; Hirata, K.; Hadgraft, J.; Lane, M.E. Influence of skin penetration enhancers on skin barrier function and skin protease activity. Eur. J. Pharm. Sci. 2014, 51, 118-122. [CrossRef]

51. Trottet, L.; Merly, C.; Mirza, M.; Hadgraft, J.; Davis, A. Effect of finite doses of propylene glycol on enhancement of in vitro percutaneous permeation of loperamide hydrochloride. Int. J. Pharm. 2004, 274, 213-219. [CrossRef]

52. Mendes, M.; Nunes, S.; Sousa, J.; Pais, A.; Vitorino, C. Expanding transdermal delivery with lipid nanoparticles: A new drug-in-NLC-in-adhesive design. Mol. Pharm. 2017, 14, 2099-2115. [CrossRef]

(C) 2020 by the authors. Licensee MDPI, Basel, Switzerland. This article is an open access article distributed under the terms and conditions of the Creative Commons Attribution (CC BY) license (http://creativecommons.org/licenses/by/4.0/). 